\title{
A NOTE ON TRANSIENT HEAT TRANSFER PROBLEMS WITH TEMPERATURE-DEPENDENT THERMAL CONDUCTIVITY AND THERMAL DIFFUSIVITY
}

\author{
R. P. S. da Gama ${ }^{a}$, \\ J. R. Cerqueirab, \\ and R. M. S. da Gamab \\ ${ }^{a}$ Pontifícia Universidade Católica \\ do Rio de Janeiro \\ Departamento de Engenharia \\ Química e Materiais \\ Rua Marquês de São Vicente 225 \\ CEP: 22451-900, Rio de Janeiro, RJ, Brazil \\ rogpaz1998@gmail.com \\ bUniversidade do Estado do Rio de Janeiro \\ Departamento de Engenharia Mecânica CEP. \\ CEP: 20550-900, Rio de Janeiro, RJ, Brazil \\ josericardoscj@gmail.com \\ rsgama@terra.com.br \\ Received: January 10, 2020 \\ Revised: February 20, 2020 \\ Accepted:May 13, 2020
}

\section{ABSTRACT}

In this work it is presented a numerical procedure for solving transient heat transfer problems in which the thermal diffusivity is strongly dependent on the temperature, with the aid of the Kirchhoff transformation associated to an usual finite difference approach. The first step consists of eliminating the nonlinear terms associated to the derivatives with respect to the position, by means of a Kirchhoff transformation, giving rise to a partial differential equation with only one nonlinear term (involving the coefficient of the derivative with respect to the time). The advance in time is carried out assuming the thermal diffusivity evaluated at a known temperature, giving rise to a semi-implicit scheme. Comparisons between this approach and the usual hypothesis are carried out in order to illustrate the effect of the dependence between the temperature and the thermal diffusivity. Some typical results are presented, based on the $(6 \mathrm{H}-\mathrm{SiC})$ Silicon Carbide properties.

Keywords: nonlinear heat transfer; temperature dependent thermal diffusivity; Kirchhoff transformation; numerical procedure

\section{NOMENCLATURE}

C specific heat

$f(x)$ initial data

$k \quad$ thermal conductivity

$L \quad$ plate thickness

$\dot{q} \quad$ heat generation per unit time and volume

$t$ time

$T$ absolute temperature

$T_{B} \quad$ temperature at the boundary

$T_{I} \quad$ initial temperature

$T_{R E F} \quad$ temperature of reference

$x \quad$ cartesian coordinate

$X \quad$ dimensionless cartesian coordinate

\section{Greek symbols}

$\alpha \quad$ thermal diffusivity

$\bar{\alpha} \quad$ thermal diffusivity of reference

$\phi \quad$ auxiliary variable

$\lambda \quad$ ratio $\alpha / \bar{\alpha}$

$\rho \quad$ mass density

$\theta \quad$ dimensionless temperature

$\tau \quad$ dimensionless time

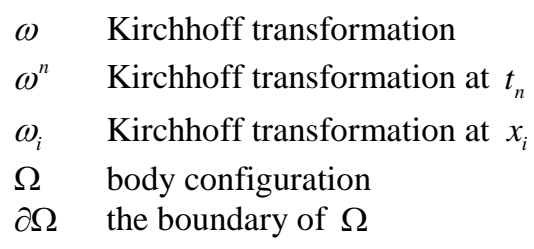

\section{INTRODUCTION}

The conduction heat transfer process in a rigid and opaque body at rest, represented here by the bounded open set $\Omega$ with boundary $\partial \Omega$, is a phenomenon whose mathematical description is very well known (Slattery, 1978; Incropera at al. 2007). The general governing equation may be written as

$$
\rho c \frac{\partial T}{\partial t}=\operatorname{div}(k \nabla T)+\dot{q}
$$

in which the quantities $\rho, c$ and $k$ depends on the temperature $T$.

Despite of representing a classical issue, these conduction heat transfer problems are, usually, treated under hypotheses that, in some cases, lead to very imprecise simulations giving rise to non dependable results. 
When a numerical simulation seems to be necessary, some hypotheses arise in order to simplify the simulation. In particular, it is usual to suppose constant thermal conductivity $k$ and constant thermal diffusivity $\alpha$.

In this work we shall consider Eq. (1) allowing that both the thermal conductivity and the thermal diffusivity may depend on the local temperature. These dependences give rise to nonlinearities.

The nonlinearity arising from the dependence of the thermal conductivity on the temperature will be surpassed with the aid of a Kirchhoff transformation, which transforms the term $\operatorname{div}(k \nabla T)$ into a linear one substituting the temperature by a new unknown. In this way, the Kirchhoff transformation eliminates the nonlinearity associated to $\operatorname{div}(k \nabla T)$ (Arpaci, 1966).

Therefore, the only nonlinearity will be the one due to the thermal diffusivity $\alpha$ (which depends on the temperature).

We shall consider Dirichlet boundary conditions (John, 1991). So, the problem focused in this work is mathematically represented by

$$
\begin{aligned}
& \rho c \frac{\partial T}{\partial t}=\operatorname{div}(k \nabla T)+\dot{q} \quad \text { in } \Omega, \quad t>0 \\
& T=\text { known on } \partial \Omega, \quad t>0 \\
& T=\text { known in } \Omega, \quad t=0
\end{aligned}
$$

In a Cartesian rectangular system of coordinates, Eq. (2) is represented by

$$
\begin{aligned}
& \rho c \frac{\partial T}{\partial t}=\frac{\partial}{\partial x}\left(k \frac{\partial T}{\partial x}\right)+\frac{\partial}{\partial y}\left(k \frac{\partial T}{\partial y}\right)+\frac{\partial}{\partial z}\left(k \frac{\partial T}{\partial z}\right)+\dot{q}, \\
& \quad(x, y, z) \in \Omega, \quad t>0 \\
& T=\text { known, } \quad(x, y, z) \in \partial \Omega, \quad t>0 \\
& T=\text { known, } \quad(x, y, z) \in \Omega, \quad t=0
\end{aligned}
$$

At this point, naturally, a question arises: Why to take into account the influence of the temperature on the thermal conductivity and on the thermal diffusivity?

In order to answer this question, let us consider a body that experiences large variations of temperature. For instance, let us consider a body made of silicon carbide. Silicon Carbide (SiC) is a semiconductor suitable for operating under high temperatures, especially when large temperature variations are present. If this body is heated from the ambient temperature until $2000 \mathrm{~K}$, are the constant thermal conductivity and the constant thermal diffusivity good assumptions?

Part of the answer (for silicon carbide $6 \mathrm{H}-\mathrm{SiC}$ ) may be obtained from Figs. 1 and 2 below, in which it is easy to see the strong dependence of $k$ and $\alpha$ on the temperature (Nilsson et al., 1997).

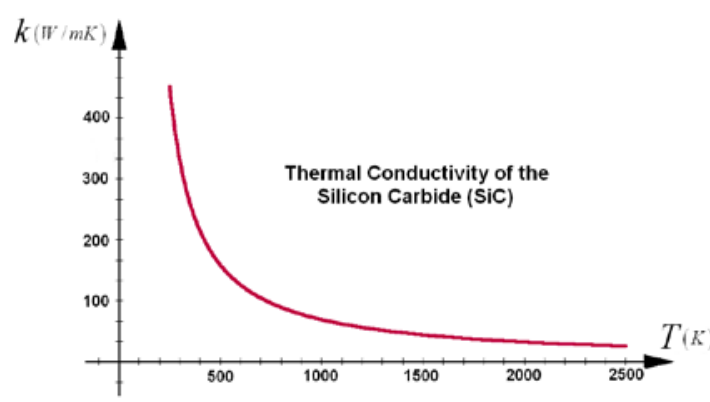

Figure 1. Thermal conductivity of the Silicon Carbide (6H-SiC ) for temperatures from $250 \mathrm{~K}$ to $2500 \mathrm{~K}$.

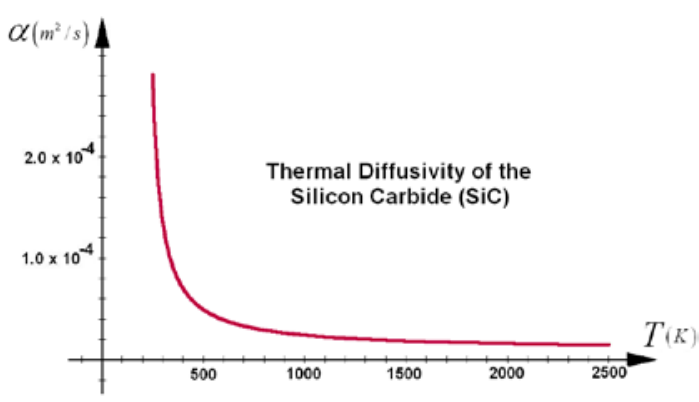

Figure 2. Thermal diffusivity of the Silicon Carbide (6H-SiC) for temperatures from $250 \mathrm{~K}$ to $2500 \mathrm{~K}$

\section{A LINEAR HEAT TRANSFER PROBLEM}

In order to illustrate the effect of the thermal diffusivity, let us consider the following problem (in which the thermal conductivity and the thermal diffusivity were assumed constant (Incropera et al, 2007)

$$
\begin{aligned}
& \frac{1}{\alpha} \frac{\partial T}{\partial t}=\frac{\partial^{2} T}{\partial x^{2}} \quad 0<x<L \\
& T=T_{B}, \quad \text { at } x=0 \text { and at } x=L \\
& T=f(x), \quad \text { for } t=0
\end{aligned}
$$

whose (exact) solution is given by (Wylie, 1975)

$$
T=\sum_{n=1}^{\infty} A_{n} \exp \left(-\alpha t\left(\frac{n \pi}{L}\right)^{2}\right) \sin \left(\frac{n \pi x}{L}\right)+T_{B}
$$

in which

$$
A_{n}=\frac{2}{L} \int_{0}^{L}\left(f(x)-T_{B}\right) \sin \left(\frac{n \pi x}{L}\right) d x
$$

This problem can be formulated in a dimensionless form with the aid of the following quantities

$$
X=\frac{x}{L} ; \quad \tau=\frac{\bar{\alpha} t}{L^{2}} ; \quad \theta=\frac{T-T_{B}}{T_{B}} \quad \text { and } \quad \lambda=\frac{\alpha}{\bar{\alpha}}
$$


Giving rise to the following problem

$$
\begin{aligned}
& \frac{1}{\lambda} \frac{\partial \theta}{\partial \tau}=\frac{\partial^{2} \theta}{\partial X^{2}} \quad 0<X<1 \\
& \theta=0, \quad \text { at } X=0 \text { and at } X=1 \\
& \theta=\frac{f(L X)-T_{B}}{T_{B}}, \quad \text { for } t=0
\end{aligned}
$$

in which $\bar{\alpha}$ is a reference diffusivity.

In this case the solution is represented as

$$
\theta=\sum_{n=1}^{\infty} a_{n} \exp \left(-\lambda(n \pi)^{2} \tau\right) \sin (n \pi X)
$$

in which

$$
a_{n}=2 \int_{0}^{1}\left(\frac{f(L X)-T_{B}}{T_{B}}\right) \sin (n \pi X) d X
$$

In Fig.3 we have the dimensionless temperature $\theta$ as a function of $\tau$, for the points $X=1 / 2$ and $X=1 / 4$, for several values of the thermal diffusivity, considering $f(x)=2 T_{B}$.
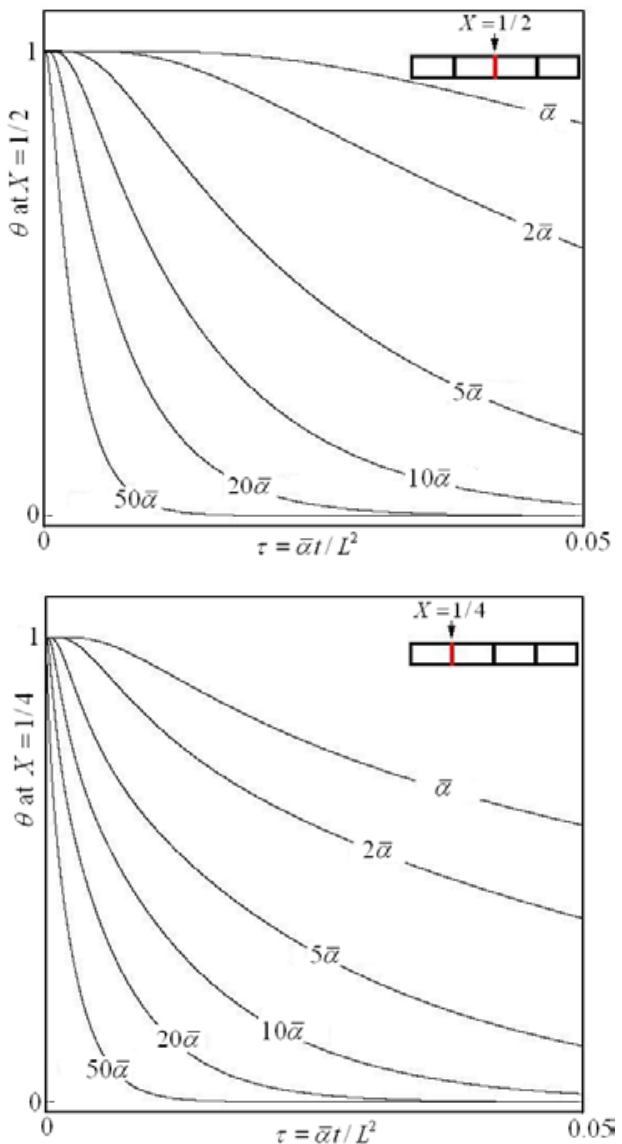

Figure 3. The temperature at $X=1 / 2$ and at $X=1 / 4$, for six different values of the thermal diffusivity, as a function of the time.
For a material with constant thermal diffusivity, the time needed for reaching a given temperature is inversely proportional to the thermal diffusivity. So, consider a material with thermal diffusivity varying from $\alpha=0.00001$ to $\alpha=0.00050$ (in some system of units). In this case, depending on the choice of the constant value for the thermal diffusivity, we may find a time 50 times greater or 50 times smaller for reaching a given temperature.

Nevertheless, this proportionality does not hold when the dependence between temperature and thermal diffusivity is strong.

These arguments lend support for considering the dependence of properties like $\alpha$ with the temperature.

\section{THE KIRCHHOFF TRANSFORMATION}

Let us introduce the Kirchhoff transformation as follows

$$
\omega=\int_{T_{R E F}}^{T} \hat{k}(\phi) d \phi, \quad k=\hat{k}(T)
$$

in which $T_{R E F}$ is a conveniently chosen temperature.

With the above definition, Eq. (2) may be rewritten as

$$
\begin{aligned}
& \rho c \frac{d T}{d \omega} \frac{\partial \omega}{\partial t}=\operatorname{div}(\nabla \omega)+\dot{q} \text { in } \Omega, \quad t>0 \\
& \omega=\int_{T_{R E F}}^{T} \hat{k}(\phi) d \phi=\text { known } \text { on } \partial \Omega, \quad t>0 \\
& \omega=\int_{T_{R E F}}^{T} \hat{k}(\phi) d \phi=\text { known } \quad \text { in } \quad \Omega, \quad t=0
\end{aligned}
$$

From Eq. (11) we have that

$$
\frac{d T}{d \omega}=\frac{1}{k}
$$

and Eq. (12) becomes

$$
\begin{aligned}
& \frac{1}{\alpha} \frac{\partial \omega}{\partial t}=\operatorname{div}(\nabla \omega)+\dot{q} \text { in } \Omega, \quad t>0 \\
& \omega=\text { known on } \partial \Omega, \quad t>0 \\
& \omega=\text { known in } \Omega, \quad t=0
\end{aligned}
$$

in which the thermal diffusivity depends on the temperature and, therefore, may be expressed as a function of $\omega$. In other words, we have

$$
\alpha=\hat{\alpha}(T)=\tilde{\alpha}(\omega)
$$

In a Cartesian rectangular system of coordinates, Eq. (14) is represented by 


$$
\begin{aligned}
& \frac{1}{\alpha} \frac{\partial \omega}{\partial t}=\frac{\partial^{2} \omega}{\partial x^{2}}+\frac{\partial^{2} \omega}{\partial y^{2}}+\frac{\partial^{2} \omega}{\partial z^{2}}+\dot{q}, \quad(x, y, z) \in \Omega, \quad t>0 \\
& \omega=\text { known, } \quad(x, y, z) \in \partial \Omega, \quad t>0 \\
& \omega=\text { known, } \quad(x, y, z) \in \Omega, \quad t=0
\end{aligned}
$$

For instance, the conductivity and the diffusivity of the Silicon Carbide are approximately given by (Nilsson et. al. 1997)

$$
\begin{aligned}
& k=\frac{61100}{T-115}, \quad 2500 K \geq T \geq 250 K \quad(W / m K) \\
& \alpha=\frac{0.0146}{T-207}, \quad 2500 K \geq T \geq 250 K \quad\left(\mathrm{~m}^{2} / \mathrm{s}\right)
\end{aligned}
$$

So, let us consider the following relationships

$$
\begin{aligned}
& k=\frac{A}{T-B}, \quad T>B \\
& \alpha=\frac{\bar{A}}{T-\bar{B}}, \quad T>\bar{B}
\end{aligned}
$$

In such cases we have

$$
\begin{aligned}
& \omega=\int_{T_{R E F}}^{T} \frac{A}{\phi-B} d \phi=A\left[\ln \left(\frac{T-B}{T_{R E F}-B}\right)\right] \Rightarrow \\
& \ln \left(\frac{T-B}{T_{R E F}-B}\right)=\frac{\omega}{A} \Rightarrow \frac{T-B}{T_{R E F}-B}=\exp \left(\frac{\omega}{A}\right) \Rightarrow \\
& T=B+\left(T_{R E F}-B\right) \exp \left(\frac{\omega}{A}\right)
\end{aligned}
$$

and, so,

$$
\alpha=\frac{\bar{A}}{B-\bar{B}+\left(T_{R E F}-B\right) \exp (\omega / A)}
$$

For the Silicon Carbide we have, assuming $T_{R E F}=250 K$

$$
\omega=61100\left[\ln \left(\frac{T-115}{135}\right)\right]
$$

And

$$
\alpha=\frac{0.0146}{-92+135 \exp \left(\frac{\omega}{61100}\right)}=\frac{0.0146}{T-207}
$$

Eq. (3) and Eq. (9) represent the same problem. Nevertheless, Eq. (9) has only one nonlinearity and does not involve any numerical approximation for the thermal conductivity. The dependence of the thermal conductivity on the temperature is embedded in the new unknown $\omega$.
Eq. (15) is illustrated in Fig. 4.

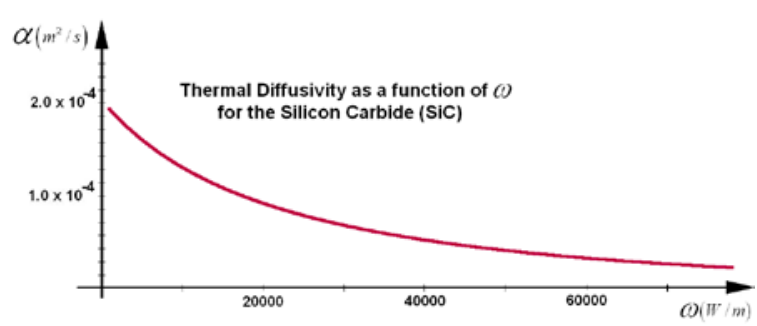

Figure 4. Thermal diffusivity of the Silicon Carbide as a function of the Kirchhoff Transformation.

\section{A SEMI-IMPLICIT ALGORITHM}

The use of the Kirchhoff transformation enables us to advance from time $t^{n}$ to time $t^{n+1}$ considering the term $\operatorname{div}(k \nabla T)$ evaluated at the time $t^{n+1}$. The only quantity evaluated at the time $t^{n}$ will be the thermal diffusivity $\alpha$.

This semi-implicit algorithm for advancing from $t^{n}$ to $t^{n+1}$ can be summarized as follows

$$
\begin{aligned}
& \frac{\omega^{n+1}-\omega^{n}}{\alpha^{n} \Delta t}=[\operatorname{div}(\nabla \omega)+\dot{q}]^{n+1} \text { in } \Omega \\
& \omega^{n+1}=\text { known on } \partial \Omega \\
& \omega^{n}=\text { known in } \Omega
\end{aligned}
$$

in which the superscript $n$ indicates that the quantity is evaluated at the time $t^{n}$.

In Eq. (23) $\alpha^{n}$ depends on $\omega^{n}$. So, the approximation at $t^{n+1}$ is obtained from a linear problem.

In a Cartesian rectangular system of coordinates, Eq. (23) is represented by

$$
\begin{aligned}
& \frac{\omega^{n+1}-\omega^{n}}{\alpha^{n} \Delta t}=\left(\frac{\partial^{2} \omega}{\partial x^{2}}\right)^{n+1}+ \\
& +\left(\frac{\partial^{2} \omega}{\partial y^{2}}\right)^{n+1}+\left(\frac{\partial^{2} \omega}{\partial y^{2}}\right)^{n+1}+\dot{q}^{n+1}, \quad(x, y, z) \in \Omega \\
& \omega^{n+1}=\text { known, } \quad(x, y, z) \in \partial \Omega \\
& \omega^{n}=\text { known, } \quad(x, y, z) \in \Omega
\end{aligned}
$$

\section{AN ONE DIMENSIONAL EXAMPLE}

In order to illustrate the proposed procedure and the effect of the thermal diffusivity, let us consider the following one dimensional problem

$$
\begin{aligned}
& \rho c \frac{\partial T}{\partial t}=\frac{\partial}{\partial x}\left(k \frac{\partial T}{\partial x}\right)+\dot{q}, \quad 0<x<L, \quad t>0 \\
& T=T_{B}, \quad \text { at } \quad x=0 \text { and at } x=L, \quad t>0 \\
& T=T_{I}, \quad 0<x<L, \quad t=0
\end{aligned}
$$


in which it was assumed that $\dot{q}$ is a known function. With the aid of the Kirchhoff transformation, Eq. (25) becomes

$$
\begin{aligned}
& \frac{1}{\alpha} \frac{\partial \omega}{\partial t}=\frac{\partial^{2} \omega}{\partial x^{2}}+\dot{q}, \quad 0<x<L, \quad t>0 \\
& \omega=\omega_{B}, \quad \text { at } \quad x=0 \text { and at } x=L, \quad t>0 \\
& \omega=\omega_{I}, \quad 0<x<L, \quad t=0
\end{aligned}
$$

in which $\omega_{B}$ and $\omega_{I}$ are obtained from $T_{B}$ and $T_{I}$ with the aid of Eq.(12).

The steady-state solution of this problem is well known (Holman, 1976), and given by

$$
\omega=\frac{\dot{q} L^{2}}{2}\left(\frac{x}{L}-\left(\frac{x}{L}\right)^{2}\right)+\omega_{B}, \quad 0<x<L
$$

or, if we consider Eq.(18) with $T_{R E F}=T_{B}$,

$$
\frac{T-B}{T_{B}-B}=\exp \left(\frac{\dot{q} L^{2}}{2 A}\left(\frac{x}{L}-\left(\frac{x}{L}\right)^{2}\right)\right), \quad 0<x<L
$$

Combining Eq. (26) with Eq.(24) we have

$$
\begin{aligned}
& \frac{\omega^{n+1}-\omega^{n}}{\alpha^{n} \Delta t}=\left(\frac{\partial^{2} \omega}{\partial x^{2}}\right)^{n+1}+\dot{q}^{n+1}, \quad 0<x<L \\
& \omega^{n+1}=\omega_{B}, \quad \text { at } \quad x=0 \text { and at } x=L \\
& \omega^{0}=\omega_{I}, \quad 0<x<L
\end{aligned}
$$

Denoting by $\omega_{i}^{n}$ the approximation for $\omega$ at the time $t^{n}$ at the position $x_{i}$, in which

$$
\begin{array}{ll}
x_{i}=(i-1) \Delta x, & \Delta x=\frac{L}{N-1} \\
t^{n+1}=t^{n}+\Delta t, \quad t^{0}=0
\end{array}
$$

we have the following finite difference equations

$$
\begin{aligned}
& \frac{\omega_{i}^{n+1}-\omega_{i}^{n}}{\alpha^{n} \Delta t}= \\
& =\frac{\omega_{i+1}^{n+1}-2 \omega_{i}^{n+1}+\omega_{i-1}^{n+1}}{(\Delta x)^{2}}+\dot{q}_{i}^{n+1}, \quad i=2,3,4, \ldots, N-1 \\
& \omega^{n+1}=\omega_{B}, \quad \text { for } i=1 \text { and for } i=N \\
& \omega^{0}=\omega_{I}, \quad i=2,3,4, \ldots, N-1
\end{aligned}
$$

Eq. (31) represents, for each $n \geq 0$, a linear system whose unknowns are $\omega_{i}^{n+1}(i=2,3, \ldots, N-1)$. This system may be conveniently rewritten as

$$
\begin{aligned}
& \omega_{i}^{n+1}=\frac{\left(\omega_{i+1}^{n+1}+\omega_{i-1}^{n+1}\right) \alpha^{n} \Delta t+\left(\dot{q}_{i}^{n+1}\right) \alpha^{n} \Delta t(\Delta x)^{2}+\omega_{i}^{n}(\Delta x)^{2}}{(\Delta x)^{2}+2 \alpha^{n} \Delta t}, \\
& \quad i=2,3,4, \ldots, N-1 \\
& \omega^{n+1}=\omega_{B}, \quad \text { for } i=1 \text { and for } i=N \\
& \omega^{0}=\omega_{I}, \quad i=2,3,4, \ldots, N-1
\end{aligned}
$$

Figure 5 presents the temperature as a function of the time at the position $x / L=0.5$, obtained with $\dot{q}=0$, for three constant values of $\alpha$ and for $\alpha$ given by Eq.(20).

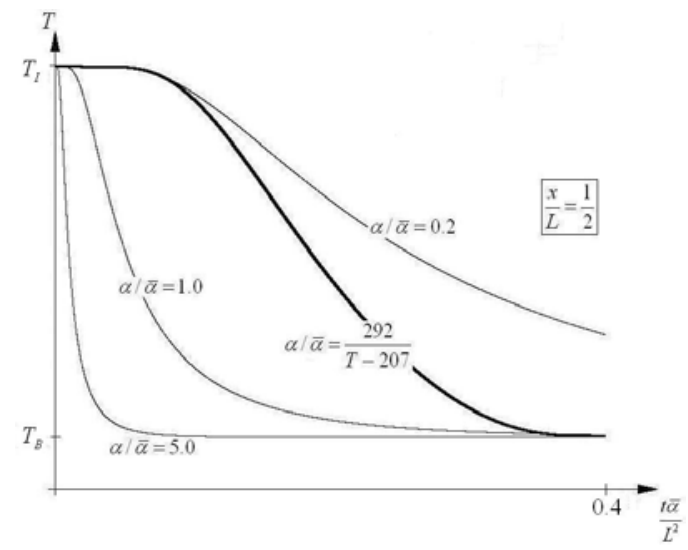

Figure 5. Temperature versus dimensionless time at $x / L=0.5$. The bold line was obtained with variable thermal diffusivity.

The bold curve was obtained taking into account the dependence between the thermal conductivity and the temperature. The quantity $\bar{\alpha}$ is the thermal diffusivity of the Silicon Carbide at $500 \mathrm{~K}\left(0.00005 \mathrm{~m}^{2} / \mathrm{s}\right)$. The maximum and the minimum thermal diffusivities within the range $250 K<T<2500 K$ are, respectively, $0.00034 \mathrm{~m}^{2} / \mathrm{s}$ and $0.000006 \mathrm{~m}^{2} / \mathrm{s}$.

Fig. 6 presents the temperature as a function of the time at the position $x / L=0.25$.

In Fig. 5 and in Fig. 6 we have $T_{B}=250 K$ and $T_{I}=2000 K$.

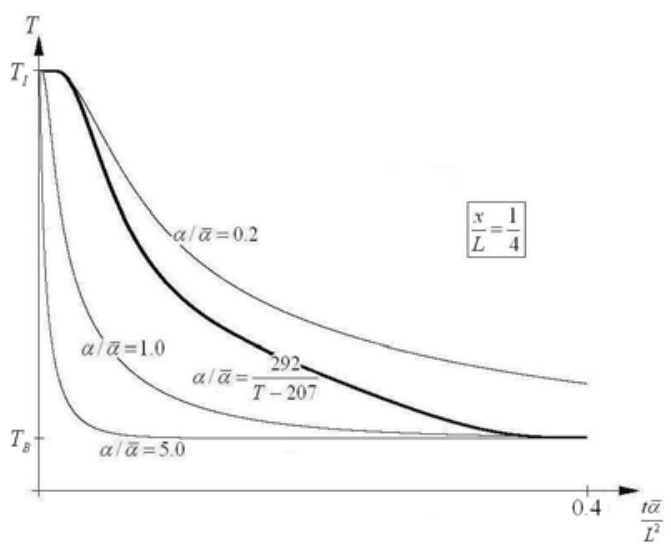

Figure 6. Temperature versus dimensionless time 
at $x / L=0.25$. The bold line was obtained with variable thermal diffusivity.
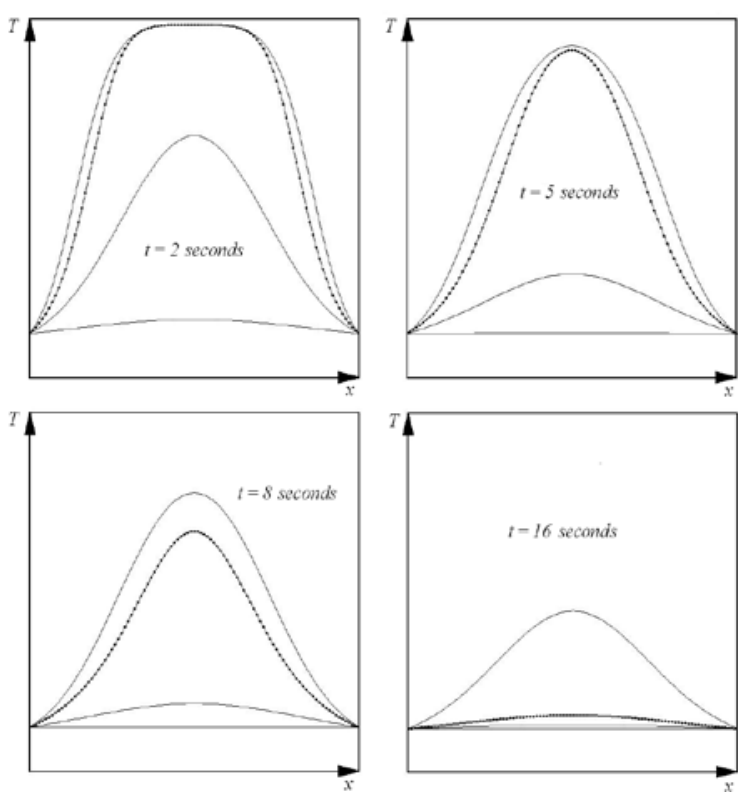

Figure 7. Temperature versus position for four different times. The line with dots shows the result obtained with variable thermal diffusivity.

In Fig.7 the considered thermal diffusivities are the same of Figs. 5 and 6. It is remarkable the different behavior in the case of variable thermal diffusivity.

Figures. 8 and 9 presents the temperature as a function of the time for three constant thermal diffusivities and for the diffusivity obtained from Eq,(22). In both we have an internal heat generation and the boundary temperature is equal to the initial one. In Fig.8 the position is $x / L=0.5$ while in Fig.9 the position is $x / L=0.25$. The bold line corresponds to Eq.(22).

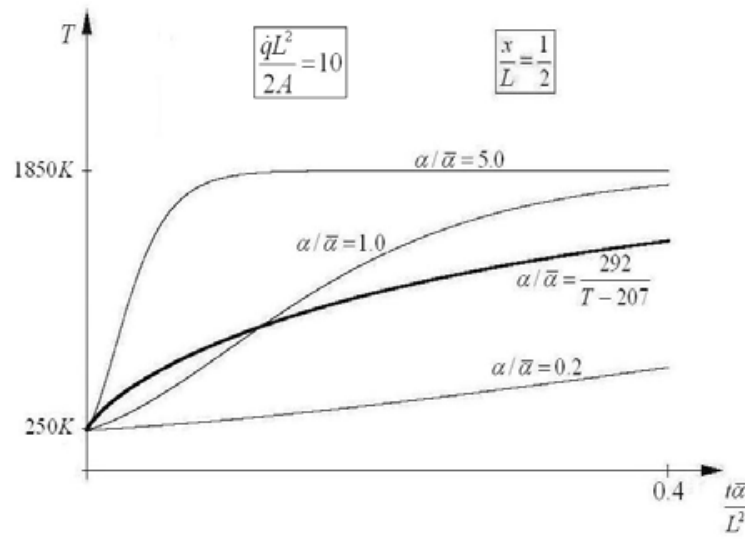

Figure 8. Temperature versus dimensionless time at $x / L=0.5$. The bold line represents the result obtained accounting for the dependence between thermal diffusivity and temperature.

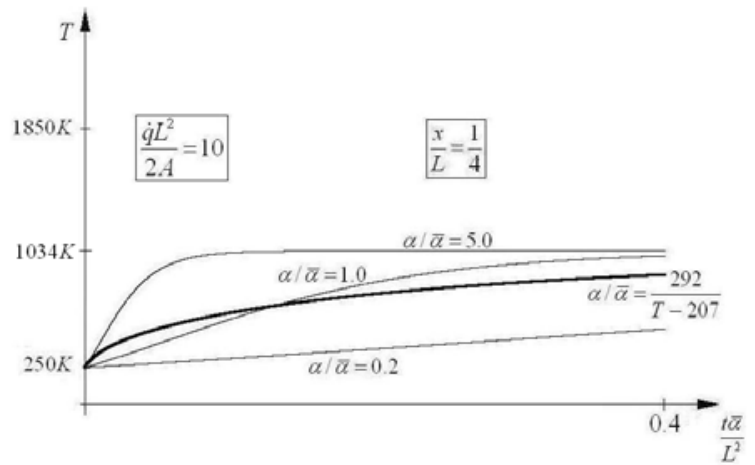

Figure 9. Temperature versus dimensionless time at $x / L=0.25$. The bold line represents the result obtained accounting for the dependence between thermal diffusivity and temperature.

The results presented in previous figures consider a constant internal heat generation. The initial and boundary temperature are the same.

Fig. 10 is similar to Fig. 8, but involves a time dependent internal heat source.

These figures illustrate the importance of accounting for the dependence between the thermal diffusivity and the temperature.

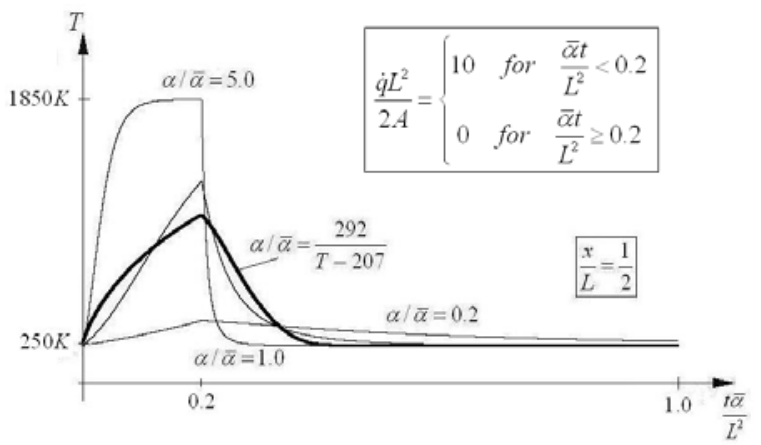

Figure 10. Temperature versus dimensionless time at $x / L=0.5$.

The bold line was obtained accounting for the dependence between thermal diffusivity and temperature. There is a time dependent heat source.

\section{CONCLUSIONS}

In this work, it was presented a protocol for simulating transient heat transfer problems taking into account the dependence of the thermal conductivity and of the thermal diffusivity with the temperature. The Silicon Carbide, which is a material widely employed when high temperatures and high temperature variations are present, was selected for carrying out numerical simulations.

The proposed semi-implicit algorithm, used for numerical simulations by means of a finite difference scheme, behaved well and seems to be a good choice for this kind of problem. 
All the presented results employed a (dimensionless) time increment $\Delta \tau=0.001$ and 101 nodes in the $x$ direction. The same simulations were carried out with 501 nodes and with $\Delta \tau=0.0005$, presenting variations less than $0.002 \%$.

It is to be highlighted that both the thermal conductivity and the thermal diffusivity were treated as temperature-dependent.

From the presented figures it is easy to see that a constant thermal diffusivity hypothesis can suggest an unrealistic time for reaching a given temperature.

\section{ACKNOWLEDGEMENTS}

The author R. M. S. Gama, gratefully acknowledge the financial support provided by the Brazilian agency CNPq (Grant 306364/2018-2).

\section{REFERENCES}

Arpaci, V. S., 1966, Conduction Heat Transfer, Addison Wesley Publishing Company. Hill.

Holman, J. P., 2002, Heat Transfer, McGraw-

Incropera, F. P., Dewitt, D. P., Bergman, T. L., and Lavine A. S., 2007, Fundamentals of Heat and Mass Transfer, 6th Edition, John Wiley \& Sons.

John, F., 1991, Partial Differential Equations, 4th Edition, Springer.

Kreyszig, E., 2005, Advanced Engineering Mathematics, 9th Edition, John Wiley \& Sons.

Nilsson, O., Mehling, H., Horn, R., Fricke, J., Hofmann, R., Muller, S. G., Eckstein, R., and Hofmann, D., 1997, Determination of the Thermal Diffusivity and Conductivity of Monocrystalline Silicon Carbide, High Temperatures-High Pressures, Vol. 29, pp.73-79.

Slattery, J. C., 1972, Momentum, Energy and Mass Transfer in Continua, McGraw-Hill Kogakusha. 